\title{
The Development of a Low Cost and Portable Light Absorbance Measurement Device for Chemical Education
}

\author{
Soranut Kittipanyangam ${ }^{\mathrm{a}}$, Kanji $\mathrm{Abe}^{\mathrm{b}}, \mathrm{Kei}_{\text {Eguchi }}{ }^{\mathrm{c}}$ \\ a,b,cFukuoka Institute of Technology, 3-30-1 Wajiro-higashi, Higashi-ku, Fukuoka, 811-0295, Japan \\ Corresponding Author: ${ }^{1}$ pokemon_ac123@gmail.com, ${ }^{2}$ s11f1002@ benefit.ac.jp, ${ }^{3}$ eguti@ fit.ac.jp
}

\begin{abstract}
This research aims to study about developing and demonstrating the low-cost and portable light absorbance measurement device for using and learning in analytical experiments. In chemistry experiments, the concentration of solutions is important for mixing the sample or making medicines. In present, a UV-spectrophotometer is commonly used to check the absorbance of solution for investigating concentration of solution by the Beer's law before bringing it to use in experiment. Although the UV-spectrophotometer has high quality, student's learning doesn't use all functions of the UV-spectrophotometer. Furthermore, the UVspectrophotometer is very expensive to buy many devices for student's learning. To support student's learning for the chemistry education, we develop a low-cost spectrophotometer by decreasing unnecessary functions and parts. The results show the coefficient of determination of light absorbance compared with the UV-spectrophotometer and the linear regression for calculating unknown concentration of solution. The effectiveness of the proposed device is demonstrated by experiments. Moreover, the program receiving the data from the proposed device can receive many data from many device by wireless network.
\end{abstract}

Keywords: Light Absorbance, UV-spectrophotometer, Beer's Law, Coefficient of Determination, Linear Regression.

\section{Introduction}

At present, the measurement of concentration of solutions is important in every analytical chemistry laboratory. Nowadays, however, there is not direct measuring method for the concentration of solutions. The measurement must use many means and converts to concentration ${ }^{(1)}$. In analytical chemistry laboratories, an
Ultraviolet-visible spectrophotometer (UV-Vis spectrophotometer), the concentration measurement device by light, is generally used to measure the light absorbance of solution. The light absorbance has relationship with the concentration of solution by Beer's law. If the light absorbance of solution is known, the concentration of solution is also known. However, the UV-spectrophotometer is expensive. Therefore, it is difficult to purchase for university's Faculty of Science. For this reason, it is not enough for every bachelor's degree students learning and making experiments.

To satisfy the scientists' requirements, we survey about the previous light absorbance devices. For example, Bano's UV-spectrophotometer used a monochromator which changes a wavelength to measure the color of solution properly in a microcontroller based spectrophotometer using a compact disc as diffraction grid ${ }^{(2)}$. This research utilized a compact disc as the monochromator to select the wavelength. And it uses the microcontroller AVR ATmega32 to calculate the light absorbance by the Beer-Lambert law. However, using the compact disc makes the device bigger than necessary. The other research is a paired emitter detector diode (PEDD) based photometry ${ }^{(3)}$ that uses one color of LED per one detector. The detector have many kinds. Each kind of detector responses to each color differently. Using the one color LED of per one detector, the device make the result accurately. However, putting all of LEDs and detectors in the device makes device bigger than necessary. Therefore, it is very difficult to create the device that can change the LEDs and detector. The conventional research reported in the 
development of a portable spectrophotometer for noncontact color measurement proposed by Liu (4), employed a photodiode array as the detector which can check absorbance of solution in many wavelengths. However, it is difficult to buy the photodiode array in general electronic stores. In a low cost LED based spectrophotometer reported in A Low Cost LED Based Spectrometer ${ }^{(5)}$, many LEDs for measuring each color of solution and a microcontroller for calculating and sending data to a computer are used. However, it is trouble to change LED, when the experiment is changed in experiments. The other research, build your own spectrophotometer ${ }^{(6)}$, used LDR as a detector. However, when the LDR receive the light until the some range of light intensity. The LDR changes resistance quickly too that data received is incorrect.

For above-mentioned reasons, we develop a light absorbance measurement device that is portable to be used outside the laboratories and fabricated with low cost for student's learning. The proposed device utilizes just visible light only. The visible spectrum is about 380-780nm. In other words, ultraviolet light is not required in the proposed device. It is known that the ultra-violet light is dangerous for human. In the proposed device, the RGB LED light is used to control wavelength by changing and mixing colors. The each color of solution has specific absorbance differently. Furthermore, some experiments cannot measure the very different concentration of solution, because the light weight is too much or too little. In the proposed device, this problem is solved by variable resistors. It changes the light weight of 3 colors of the LED. Since the LED can change light weight, the proposed device can measure many periods of concentration of solution. In addition, the proposed device can send data to a computer and save them in Microsoft Excel via RS232 or Xbee that easy to collect the data.

\section{Principle of analysis}

\subsection{Light Absorbance}

In chemistry experiment, light absorbance is the common logarithm of the ratio of incident to transmit radiant light through a material. Fig.1. illustrates the process of the light absorbance. When the light goes through the sample, the solution absorbs one part of the light.

To measure the concentration of solutions, measuring light absorbance is general method in analytical science laboratory. In experiment, the measurement is used the color light which the solution can absorbs best. The solution

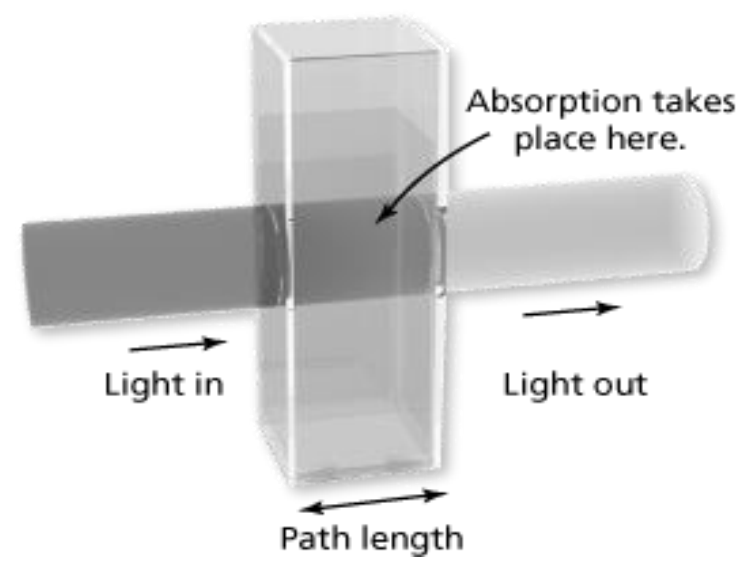

Fig. 1. Light Absorbance.

Table 1. Light absorbance.

\begin{tabular}{|l|l|l|}
\hline Wavelength & Absorbance color & Color of solution \\
\hline $380-435$ & Violet & Green-yellow \\
\hline $435-480$ & Blue & Yellow \\
\hline $480-490$ & Blue-green & Orange \\
\hline $490-500$ & Green-blue & Red \\
\hline $500-560$ & Green & Violet \\
\hline $560-580$ & Green-yellow & Violet \\
\hline $580-595$ & Yellow & Blue \\
\hline $595-650$ & Orange & Blue-green \\
\hline $650-780$ & Red & Green-blue \\
\hline
\end{tabular}

absorbs every color of light, but each color of solution has specific absorbance differently. If the experiment uses the light which the solution absorbs hardly, the result is observed hard or mistake. Table 1 shows the relation between the color of solution, wavelength period of color of light and the color of light which the solution can absorb best. For example, red solution can absorb green-blue light or a light of 490-500nm best and yellow solution can absorb blue light or a light of 435-480nm best.

In spectroscopy, the absorbance of a material is a logarithmic ratio of the amount of radiation falling upon a material to the amount of radiation transmitted through the material. If the solution is high concentration, the solution is also high absorbance. Absorbance measurements are often carried out in analytical chemistry. In UV-spectrophotometer, it is used for analytical chemistry of experiment.

\subsection{Beer's law}

Beer's law or Beer-Lambert law in (1) relates the attenuation of light to properties of the material which the 
light is traveling. The law is commonly applied to chemical analysis measurements and used in understanding attenuation in physical optics.

$$
A=-\log \frac{I}{I_{0}}=\varepsilon c l
$$

In (1), the absorbance, $A$, is directly related to the concentration, $c$, of the compound, the path length of the sample, $l$, and the molar absorption coefficient, $\varepsilon$, which is a wavelength-dependent constant characteristic of the compound. $I_{0}$ is the incident light intensity and $I$ is the transmitted light intensity [5].

$$
\frac{I}{I_{0}}=\frac{I_{\text {sample }}}{I_{\text {solvent }}}=\frac{V_{\text {sample }}-V_{\text {zero }}}{V_{\text {solvent }}-V_{\text {zero }}}
$$

In measurement, the detector doesn't give absorbance value directly. The detector gives information as a voltage. Therefore, the calculation equation must use the relation between light intensity and voltage. However, (1) uses incident light intensity and transmitted light intensity. For this reason, $I$ and $I_{0}$ are replaced the light intensity transmitting sample, $I_{\text {sample }}$, and the light intensity transmitting, $I_{\text {solvent }}$. The conversion of light intensity to the voltage is performed by using the relationship between incident the light and voltage. $V_{\text {sample }}$ is voltage when the light transmits a sample. $V_{\text {solvent }}$ is voltage when the light transmits a solvent. However, the equation (1) is not assumed that circuit sends out $0 \mathrm{~V}$ when no light falls on the detector, and make the correction by subtracting the voltage at zero light ( $\left.V_{\text {zero }}\right)$. The detector exhibits a linear relationship between the incident light and the voltage ratio described in (2) ${ }^{(4)}$. The absorbance is obtained by substituting (1) and (2) into (3).

$$
A=-\log \frac{V_{\text {sample }}-V_{\text {zero }}}{V_{\text {solvent }}-V_{\text {zero }}}
$$

Beer's law uses a logarithm in equation, so light absorbance value is in the range of 0 to 2 .

\subsection{Coefficient of Determination}

In statistics, the coefficient of determination, denoted $R^{2}$ (the square of the correlation coefficient), is a number that indicates how well data fit a statistical model whose main purpose is either the prediction of future come or the testing of hypotheses, on basic of other related information. It provides a measure at how well observed outcome is replicated by the model, as the proportion of total variation of outcomes.

$$
R^{2}=\left\{\frac{\sum_{i=1}^{n}\left(x_{i}-\bar{x}\right)\left(y_{i}-\bar{y}\right)}{\sqrt{\sum_{i=1}^{n}\left(x_{i}-\bar{x}\right)^{2} \sum_{i=1}^{n}\left(y_{i}-\bar{y}\right)^{2}}}\right\}^{2}
$$

The coefficient of determination in (4) is the relation of values which is during 0 to 1 . If the coefficient of determination is close to 1 , it shows the good relationship.

The experiment uses the coefficient of determination to check value of relationship between light absorbance and concentration of solution by number of solution, $n$, concentration of solutions, $x_{i}$, mean of concentration of solution, $\bar{x}$, light absorbance, $y_{i}$, and mean of light absorbance, $\bar{y}(8)$

\subsection{Linear Regression}

In statistics, the linear regression is an approach for modeling the relationship between a scalar dependent variable $y$ and one or more explanatory variables (or independent variable) denoted $X$.

$$
Y=a+b X
$$

In experiment uses the linear regression (5) is used for finding unknown concentration of solution. $Y$ is concentration of solution and $X$ is light absorbance. In (5), $a$ is constant value when $X$ is 0 in $Y$ axis and $b$ is slope ${ }^{(8)}$.

\section{Circuit configulation}

Fig.2 shows the proposed device and preparing experiment that it has the purposed device and the 3 colors (Green, Blue, and Red) of 5 concentration of solution. After the measurement of each color is finished, user brings every absorbance of color of solutions to calculate coefficient of determination and compare with the result of the coefficient of determination of the general portable spectrophotometer. If the experiments have many concentration of solution, the coefficient of determination is correct.

Fig.3 illustrates the circuit configuration of the propose device. Many parts are removed many parts for lighter and

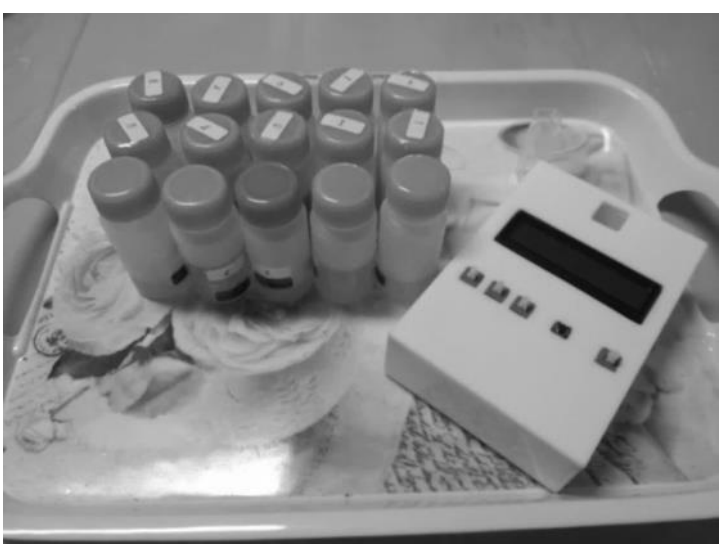

Fig. 2. Proposed light absorbance measurement device 


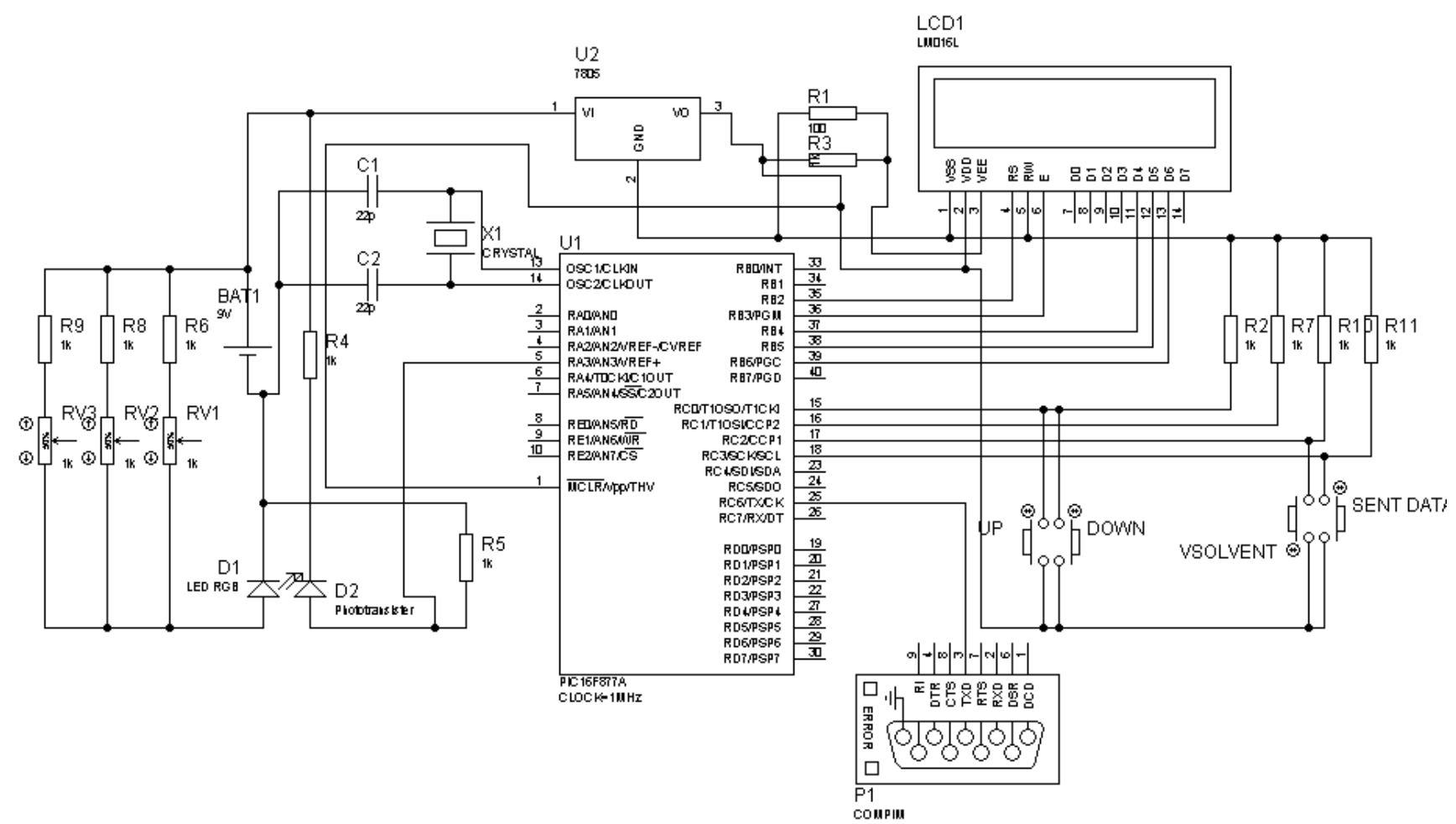

Fig. 3. Circuit configuration

smaller than the UV-spectrophotometer. The power supply is a battery $9 \mathrm{~V}$ to turn on LEDs. IC7805 is used to regulate $9 \mathrm{~V}$ to $5 \mathrm{~V}$ for using a microcontroller and interface Xbee. The light source is the RGB LED which can change wavelength and light weight by the input voltage (using variable resistor) of 3 colors of LEDs. The monochromator is removed from the proposed device, because the LED can change colors. The photo detector is photo transistor which gives results stable more than LDR and the photodiode. Therefore, in experiment, it gives data comparing with the general spectrophotometer better result than LDR and photodiode.

To calculate the Beer's law, the microcontroller PIC16F877A is used, where the program in the microcontroller is applied from the voltage meter. This proposed device shows the data of absorbance results by LCD and sends data to a computer by Xbee or comport.

\subsection{Price of Device}

The proposed device is assembled for chemical experimental learning; so many devices are necessary for students. Table 2 shows the parts list of the proposed device. The proposed device can be assembled by about 2300 JPY.

To save the light absorbance data from the proposed device, the interface is essential. For this reason, we propose
2 kinds of interfaces for sending data to a computer. The first is RS232 used mostly. The RS232 is very simple to send data to a computer and cheap for about 940 JPY. Table 3 shows the cost of RS232.

Another interface is Xbee. It is wireless interface produced by Digi International. It can create wireless network for sending data to a computer by many devices at the same time. It is more comfortable than RS232. It can move and send data to a computer in network area but RS232 must connect to computer currently for sending data. Xbee is easier than RS232 for sending data but it is more expensive than RS232. The master device receiving data from slave devices is about 1700JPY. The slave device sending data to master device is about $3700 \mathrm{JPY}$ for one device. Table 4 shows cost of one master and a master-slave set.

\section{Operation}

\subsection{Measurement Part}

The proposed device consists of a RGB LED, variable resistors, a photo transistor, resistor, and a battery $9 \mathrm{~V}$. LED RGB as light source can change colors and light weight by switches and variable resistors. When the LED RGB holds on the solution against a light, the solution absorbs one part of the light as shown in Fig.1. The light, that the solution 
cannot absorb, transmits the sample to photo transistor. The photo transistor that receives the light decreases resistance itself. The resistance of the photo transistor determines the input voltage. The input voltage is divided by voltage divider so that maximum input doesn't exceed $4.5 \mathrm{~V}$, before sending it to calculate in the microcontroller PIC16F877A.

\subsection{Calculation Part}

Input voltage from measurement part is sent to the microcontroller PIC16F877A. The program of the microcontroller is applied from a voltage meter. The $\mathrm{V}_{\text {solvent }}$ button is used to memorize $\mathrm{V}_{\text {solvent }}$ in Beer's law. This button is used for one light of one sample. For example, red solution can absorb green-blue light. The green light is used first and

Table 2. Proposed light absorbance measurement device

\begin{tabular}{|l|r|r|}
\hline Part name & Number & Price(JPY) \\
\hline PIC16F877A & 1 & 550 \\
\hline Battery box & 1 & 110 \\
\hline IC7805 & 1 & 40 \\
\hline LCD16×2 & 1 & 900 \\
\hline RGB LED & 1 & 200 \\
\hline Phototransistor & 1 & 20 \\
\hline Resistor 1k $\Omega$ & 10 & 1 \\
\hline Variable resistance & 3 & 40 \\
\hline Capacitor 22pF & 1 & 2 \\
\hline Switch 1 & 4 & 10 \\
\hline Switch 2 & 3 & 50 \\
\hline Battery 9V & 1 & 100 \\
\hline Crystal 4MHz & 1 & 30 \\
\hline \multicolumn{1}{|c|}{ Total } & 27 & 2272 \\
\hline
\end{tabular}

Table 3. Parts list of the interface by RS232

\begin{tabular}{|l|r|r|}
\hline Part name & \multicolumn{1}{|c|}{ Number } & \multicolumn{1}{|l|}{ Price(JPY) } \\
\hline D-sub 9 connector Pin out & 1 & 40 \\
\hline USB to RS232 serial port 9 Pin DB9 & 1 & 900 \\
\hline Total & 2 & 940 \\
\hline
\end{tabular}

Table 4. Parts list of the interface by Xbee

\begin{tabular}{|l|r|r|}
\hline Part name & Number & \multicolumn{1}{|c|}{ Price(JPY) } \\
\hline Xbee module XB-24-Z7PIT-004 & 2 & 1700 \\
\hline Xbee socket 2.54mm & 1 & 300 \\
\hline Xbee USB-serial converter & 1 & 1280 \\
\hline Micro USB (A-MicroB)cable & 1 & 458 \\
\hline \multicolumn{1}{|c|}{ Total } & 5 & 5438 \\
\hline
\end{tabular}

next blue. When the solvent is pushed to the holder, the button solvent must be pushed to memorize the $V_{\text {solvent }}$ of green light of red solution. After the measuring of red solution by green light is finished, the light is changed to blue. The $\mathrm{V}_{\text {solvent }}$ button must be pushed again to memorize $\mathrm{V}_{\text {solvent }}$ of blue light of red solution. The sent data button is used for sending data to a computer by text file shown in Fig. 4.

\subsection{Program}

Program is Microsoft Excel. It receives, save and list text file data calculated by microcontroller by Xbee or RS232. Fig.4 shows the text file sent from the device. At one time, 7 characters are sent from device. The first two characters denote the device number or solution number which can change by buttons up and down for choosing the number. The next five characters are the data of a light

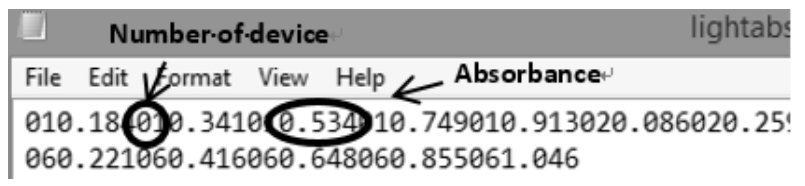

Fig. 4. Text files from device

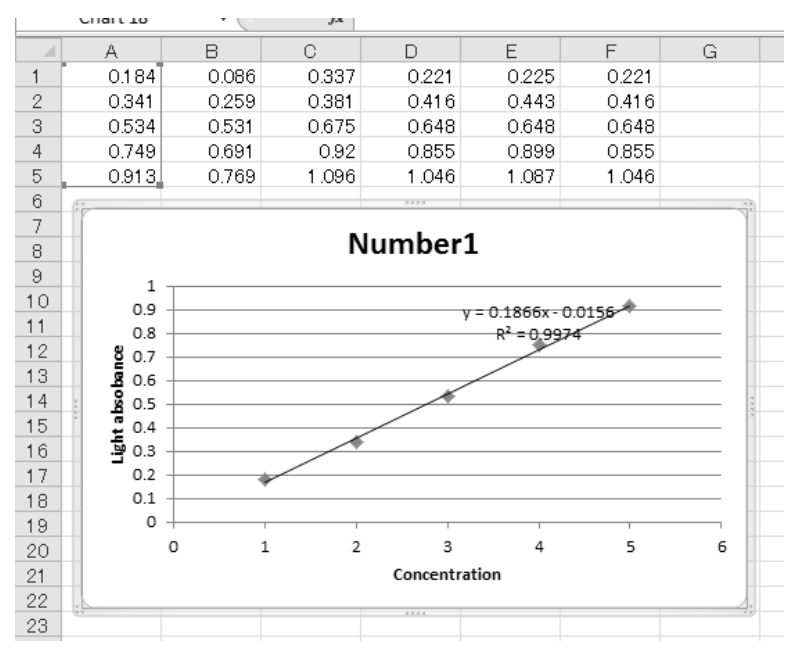

Fig. 5. Microsoft Excel Program

Table. 5. Text files from device

\begin{tabular}{|c|c|}
\hline 1 & violet \\
\hline 2 & blue \\
\hline 3 & blue-green \\
\hline 4 & green \\
\hline 5 & yellow \\
\hline 6 & red \\
\hline
\end{tabular}


absorbance. The number of device decides the column which the light absorbance put in the cell. The data from many devices are listed in Microsoft Excel as shown in Fig.5. Moreover, program can create graph and calculate coefficient of determination and linear regression by checking 1 button only showing in Fig.5.

\section{Experimental result}

In the experiment concerning the proposed device of Fig.2, the 3 colors of solutions that are red, green and blue of 5 concentrations were used. The more experiment has concentration of solution, the more coefficient of determination is correct. The five or six concentrations of solution are used in this experiment. The experiment finds the light absorbance from concentration of solution. First, the experiment finds the best color light of the proposed device and best wavelength of spectrophotometer which solution absorbing best by using every color and wavelength. The best color of the proposed device value and the best wavelength of spectrophotometer value are brought to calculate to find the coefficient of determination and linear regression and comparing efficiency and error between UVspectrophotometer and the proposed device. In an ideal case, the coefficient of determination is 1 , if each concentration of solution increases steadily. The graph from proposed device in Fig. 7, 10 and 13, the color of axis y is following in table 5. The lower line is the least concentration.

\subsection{Red Solution}

In Fig. 6, it is result of the light absorbance of red solution in many wavelength by spectrophotometer. The graph shows that the light absorbance is over $600 \mathrm{~nm}$ cannot be absorbed or can absorbed a little bit. The wavelength at over $600 \mathrm{~nm}$ is mixed the red color, so the result shows that the red solution cannot absorb red light. Furthermore, the graph shows that the red solution can absorb the light about $500 \mathrm{~nm}$ best which this color of wavelength is green-blue.

In Fig. 7 is result of red solution from the proposed device. It shows that the red solution can absorb color number 2-4 best which they are green-blue color period.

In Fig. 8, the best light absorbance from Fig. 6 and 7 are brought to plot the graph for comparing. It shows the coefficient of determination and linear regression of the spectrophotometer and proposed device. The result from spectrophotometer uses a light of $490 \mathrm{~nm}$. The proposed device uses a green-blue light. The spectrophotometer's

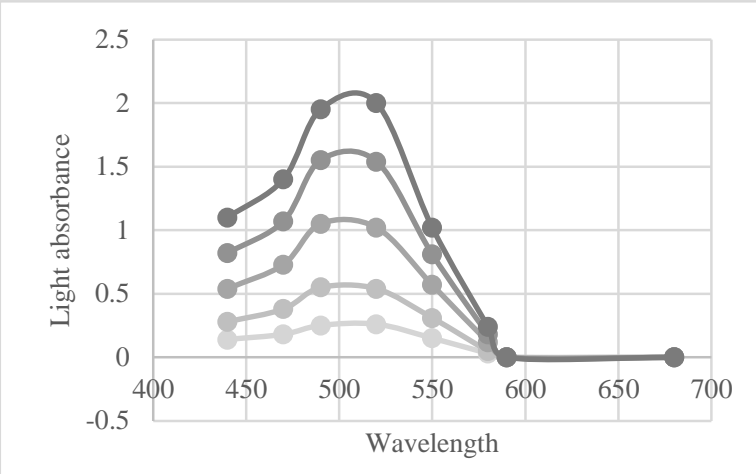

Fig. 6. Result of red solution from spectrophotometer

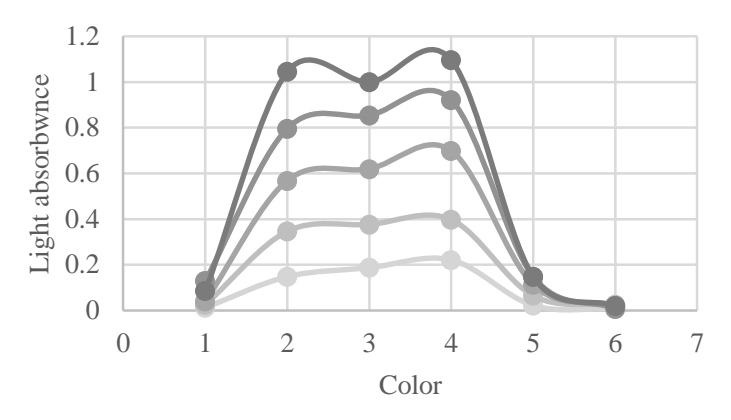

Fig. 7. Result of red solution from proposed device

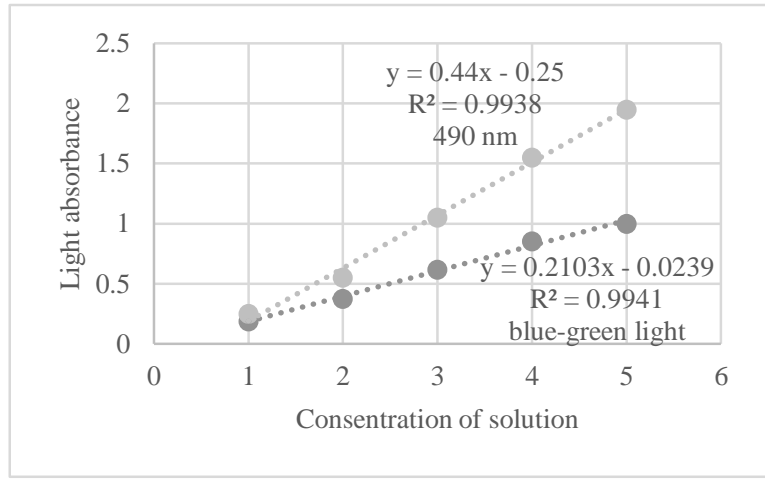

Fig. 8. Red solution result compare with spectrophotometer and proposed device

coefficient of determination is 0.9938 and the proposed device's coefficient of determination measured is 0.9941 . The error between the proposed device and UVspectrophotometer is $0.03 \%$.

\subsection{Green Solution}

In Fig. 9, it is result of the light absorbance of green solution in many wavelength by spectrophotometer. The graph shows that the light absorbance period 500-550nm 


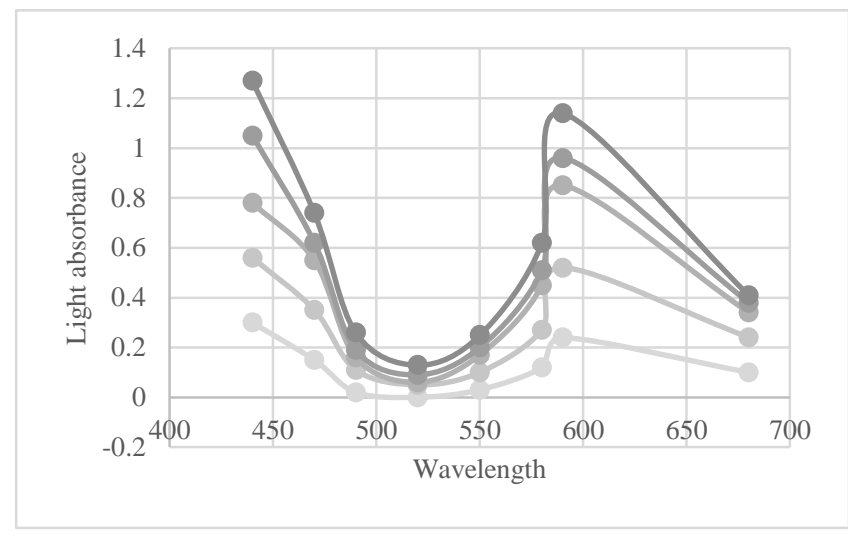

Fig. 9. Result of green solution from spectrophotometer

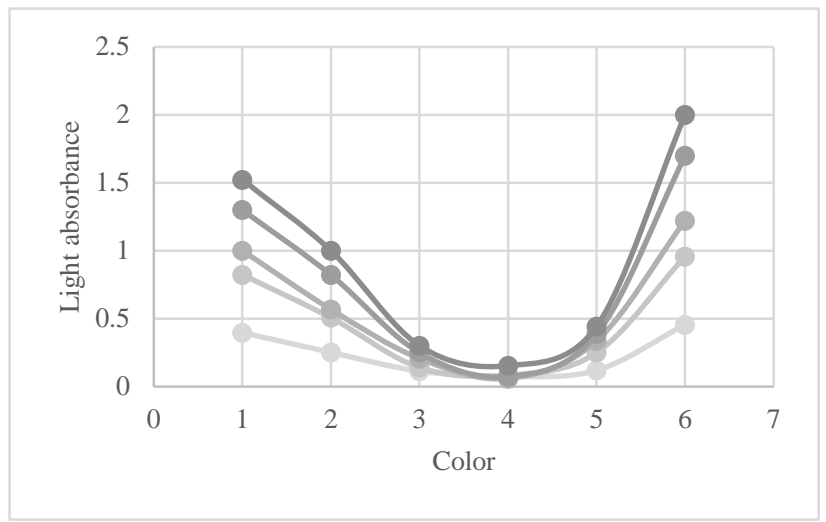

Fig. 10. Result of green solution from proposed device

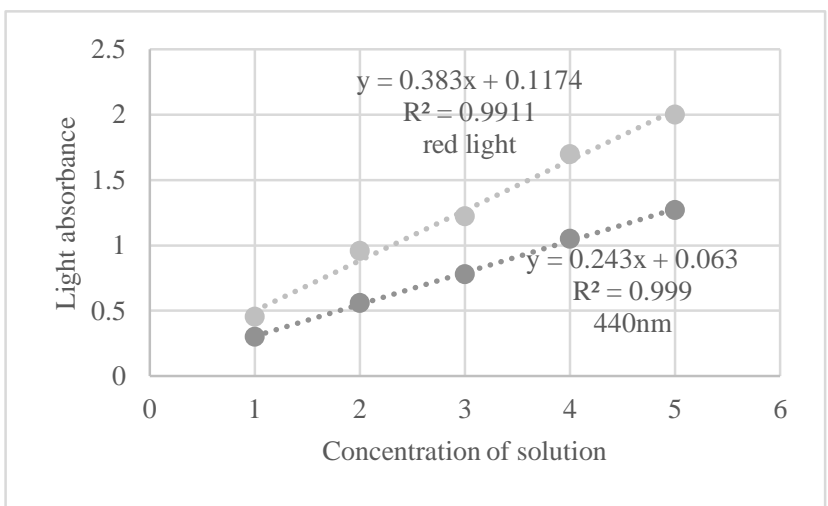

Fig. 11. Green solution result compare with spectrophotometer and proposed device

cannot be absorbed or can be absorbed a little bit. The wavelength is in period 500-550nm is green, so the result shows that the green solution cannot absorb green light. Furthermore, the graph shows that the green solution can absorb the light about $450 \mathrm{~nm}$ and $600 \mathrm{~nm}$ best which this color of wavelength is blue and orange.

In Fig. 10 is result of green solution from the proposed device. It shows that the green solution can absorb color number 1 and 6 best which they are violet and red color. Fig. 9 and 10 shows that the green solution absorbs the color mixed red and blue.

In Fig. 11, the best light absorbance is brought to plot the graph. It shows the coefficient of determination and linear regression of the spectrophotometer and proposed device. The result from spectrophotometer uses a light of $440 \mathrm{~nm}$. The proposed device uses a red light. The spectrophotometer's coefficient of determination is 0.999 and the proposed device's coefficient of determination measured is 0.9911 . The error between the proposed device and UV-spectrophotometer is $0.79 \%$.

\subsection{Blue Solution}

In Fig. 12, it is result of the light absorbance of blue solution in many wavelength by spectrophotometer. The graph shows that the light absorbance is less 500nm cannot be absorbed or can absorb a little bit. The wavelength less $500 \mathrm{~nm}$ is blue, so the result shows that the green solution cannot absorb green light. Furthermore, the graph shows that the blue solution can absorb the light about $600 \mathrm{~nm}$ best which this color of wavelength is yellow and orange.

In Fig. 13 is result of blue solution from the proposed device. It shows that the blue solution can absorb color number 1 and 6 best which they are violet and red color. Fig 12 and 13 show that the blue solution absorbs the color mixed green-red.

In Fig. 14, the best light absorbance is brought to plot the graph. It shows the coefficient of determination and linear regression of the spectrophotometer and proposed device. The result from spectrophotometer uses a light of $590 \mathrm{~nm}$. The proposed device uses a red light. The spectrophotometer's coefficient of determination is 0.9955 and the proposed device's coefficient of determination measured is 0.9996. The error between the proposed device and UV-spectrophotometer is $0.41 \%$.

\section{Conclusions}

The portable spectrophotometer for chemical education has been proposed in this paper. It solves many problems to create the low cost absorbance device. The proposed device is created easily for chemical learning. It is easy to understand the circuit in the device and afforded for general part. To replace UV-spectrophotometer for education, the scientist needs the coefficient of determination more than 0.99XX. In an ideal case, UV-spectrophotometer's 


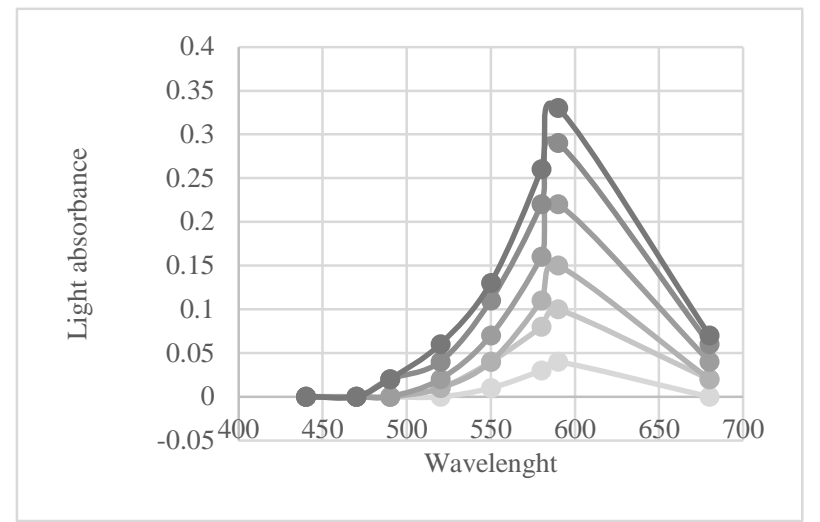

Fig. 12. Result of blue solution from spectrophotometer

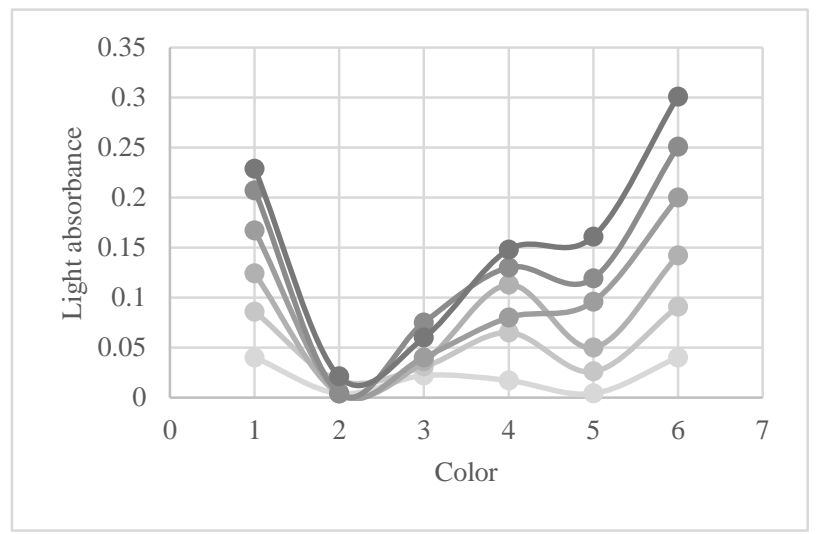

Fig. 13. Result of blue solution from proposed device

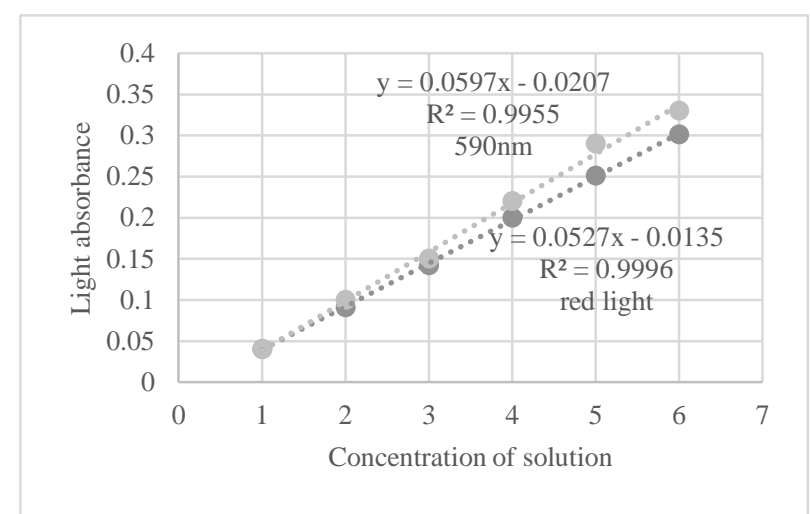

Fig. 14. Blue solution result compare with spectrophotometer and proposed device

concentration of solution is 1. The proposed device's concentration of solution is must more than $0.99 \mathrm{XX}$, so the error of proposed device comparing with spectrophotometer must be less than $1 \%$.From the experimental results of 3 colours, the error of the proposed device comparing with the spectrophotometer is less than $1 \%$. It shows the efficiency of the proposed device is near the general spectrophotometer more than $99 \%$. So the proposed device can be replaced with spectrophotometer for learning. The proposed device is cheaper than general portable spectrophotometer. It is created for about 2300 JPY and interface RS232 940 JPY. Total is about 3140 JPY only.

\section{Discussion}

In experiment, we developed the proposed device and compare with the conventional device for checking the efficiency of the proposed device. The conventional device's light source is LED which know light of wavelength. The more it has wavelength, it is expensive. In the other hand, the proposed device's light source is LED RGB. It doesn't know wavelength when the colors of light are mixed. In this point, the conventional device is better than the proposed device if the scientist need the details. However, the proposed device is created for chemical education which the user has a knowledge about relationship between the color of light and the wavelength of light. It is not serious problem for education. If this proposed device use many LED that is known the light of wavelength. Its efficiency is same as the conventional device.

\section{References}

(1) C. Areejitranusorn "Scientific instrument" Bangkok, 2001, pp.227-271.

(2) S. Bano, T. Altaf, and S. Akbar, "Microcontrolled Based Spectrophotometer Using Compact Disc As Diffraction Grid”,_IEEE, 2010, pp.332-336

(3) L. Tymecki, M. Pokrzywnicka and R. Koncki, "Paired emitter detector diode(PEDD)-based photometryalternative approach", The Royal Society of Chemistry, 2008, pp.1501-1504

(4) S.M. Liu, "The Development of a Portable Spectrophotometer for Noncontact Color Measurement”, IEEE, 2004, pp.155-162

(5) T. Yeh and S. Tseng, "A Low Cost LED Based Spectrometer" Department of Electronic Engineering. Fortune Institute of Technology, Kaohsiung, Taiwan, R.O.C. 2006

(6) S. J. Tavener and J. E. Thomas-oates," Build your own spectrophotometer" EDUCATION IN CHEMISTRY, pp.151-154,2007

(7) Smart learning .2012. Advance PIC Microcontroller in C. Bangkok: Smart learning

(8) M. Tiantong, "Statistics and Research Methods in Information Technology”, pp.363-386 